\title{
Reliability of Plasma Von Willebrand Factor Antigen in Prediction of Esophageal Varices in Patients with Liver Cirrhosis
}

\author{
Hasan Sedeek Mahmoud1, Ali A. Ghweil1, Shamardan E. Bazeed1, Hanan M. Fayed², \\ Mona M. Abdel Meguid2 \\ ${ }^{1}$ Department of Tropical Medicine and Gastroenterology, Faculty of Medicine, South Valley University, Qena, \\ Egypt \\ ${ }^{2}$ Department of Clinical Pathology, Faculty of Medicine, South Valley University, Qena, Egypt \\ Email: hasan sedeek@yahoo.com
}

Received 17 May 2015; accepted 12 June 2015; published 15 June 2015

Copyright (C) 2015 by authors and Scientific Research Publishing Inc.

This work is licensed under the Creative Commons Attribution International License (CC BY). http://creativecommons.org/licenses/by/4.0/

(c) (i) Open Access

\begin{abstract}
Background: Bleeding esophageal varices (OVs) due to portal hypertension are one of the major complications with high mortality in liver cirrhosis. So, early detection and management are mandatory. Aim: To evaluate the role of Von Willebrand factor (VWF) in predicting the presence of OVs. Patients and Methods: 62 patients with liver cirrhosis representing different Child-Pugh classes were included. The diagnosis of liver cirrhosis was based on the combination of clinical, laboratory and US examinations. All included patients underwent the following investigations: complete blood count, liver function tests (ALT, AST, serum bilirubin, albumin and total protein, prothrombin time (PT) and concentration (PC), INR and serum alkaline phosphatase), serum creatinine, Von Willebrand factor antigen (VWF-Ag) measurement and abdominal US. Upper endoscopic evaluation was done to detect presence or absence of varices (esophageal or gastric) and/or PHG. Results: 38 males and 24 females with their mean age (46 \pm 12 years old) were included. Plasma Von Willebrand factor-Ag level was significantly higher in patients with OVs than those without varices $(P$ value $=0.000)$. Also, its level was significantly higher in patients with higher grade of OVs, G3 than those with G1 or G2 (P value $=0.000)$. Patients with large OVs including those with G2 and G3 showed significantly higher values of VWF than those with small OVs (NO and G1) (P value $=0.000$ ). $\mathrm{VWF}$ was independent predictor for detecting the presence of OVs with good sensitivity (90), specificity (77.3) and accuracy (85.5) at a cutoff value of $1.74 \mathrm{U} / \mathrm{ml}$. Also it was an independent predictor for detecting the presence of large OVs with good sensitivity (91.2), specificity (85.7) and accuracy (88.7) at a cutoff value of $2.16 \mathrm{U} / \mathrm{ml}$. Conclusion: VWF-Ag could be used as a non invasive laboratory independent predictor for the detection of OVs.
\end{abstract}

How to cite this paper: Mahmoud, H.S., Ghweil, A.A., Bazeed, S.E., Fayed, H.M. and Meguid, M.M.A. (2015) Reliability of Plasma Von Willebrand Factor Antigen in Prediction of Esophageal Varices in Patients with Liver Cirrhosis. Open Journal of Gastroenterology, 5, 49-57. http://dx.doi.org/10.4236/ojgas.2015.56010 


\section{Keywords}

\section{Von Willebrand Factor Antigen, Esophageal Varices, Prediction}

\section{Introduction}

Portal hypertension (PHT) is the most important consequence of cirrhosis and its presence is a hard endpoint for clinically relevant outcomes in terms of varices, ascites, hepatorenal syndrome, and encephalopathy [1]. Variceal hemorrhage is a major cause of mortality in patients with portal hypertension [2].

Searching for noninvasive biomarkers for PHT is of considerable interest; VWF, P-selectin, and 8-iso-PGF2a have been identified as surrogate markers of endothelial dysfunction and levels of these factors are increased in patients with cirrhosis compared with controls. In patients with PHT, VWF levels significantly correlated with HVPG, Child-Pugh, and MELD scores. In addition, peripheral VWF levels with a cut-off value of $216 \mathrm{U} / \mathrm{dL}$ (Youden index) were also predictive of clinical outcomes (PHT-related events and liver transplantation) [3].

VWF is a large multimeric protein with a crucial role in primary hemostasis in healthy subjects, as evidenced by the severe bleeding tendency associated with complete VWF deficiency [4]. Conversely, elevated levels of VWF are associated with arterial thrombosis [5].

Because VWF-Ag plays a crucial role in primary hemostasis and is an indicator of endothelial activation and development of thrombotic vascular obliteration, these are considered as possible mechanisms leading to PHT [6].

Previous studies in patients with liver cirrhosis and portal hypertension reported that: plasma level of VWF$\mathrm{Ag}$ is significantly higher with more progression and impairment of the condition and in patients with esophageal varices and in patients with clinically significant PHT (hepatic venous pressure gradient $=H V P G \geq 10$ mmHg) [7] [8].

\section{Aim of the Work}

To assess the level of plasma VWF-Ag in cirrhotic patients with and without OVs and to evaluate its role in predicting the presence of OVs and large varices.

\subsection{Patients and Methods}

62 patients with liver cirrhosis representing different Child-Pugh classes were included in the current study. The diagnosis of liver cirrhosis was based on the combination of clinical, laboratory and US examinations. Blood pressure, pulse rate and body temperature measurements for all included patients were in normal ranges. Patients with possible diseases which may be responsible for high VWF Ag levels including; cardiac failure, diabetes, hypertension, hyperlipidemia, renal failure, pregnancy and patients with infectious symptoms at the time of sampling, were excluded. Patients with malignancy, patients on physical training or under IFN therapy and patients refused to participate in the study were also excluded.

Methods: all included patients in this cross sectional study underwent the following investigations: complete blood count, liver function tests (ALT, AST, serum bilirubin, albumin and total protein, prothrombin time (PT) and concentration (PC), INR and serum alkaline phosphatase) and serum creatinine. Abdominal US was done to confirm the diagnosis of liver cirrhosis; detect the size of the liver and the spleen and measure the diameter of the portal and splenic vein. Upper endoscopic evaluation (using Olympus, GIF-XQ260 instrument) was done for all included patients. Presence or absence of varices (esophageal or gastric) and/or PHG were detected. The size of OVs was graded as described by Beppu et al. (1981) into grade 1: enlarged but straight varices, grade 2: enlarged tortuous varices and grade 3: coiled shaped markedly enlarged varices [9].

Laboratory work: About $7 \mathrm{ml}$ blood were obtained from each patient by venipuncture; two $\mathrm{ml}$ on EDTA tube for CBC (using Sysmex KX21; Sysmex corporation), $1.8 \mathrm{ml}$ on 3.2\% sodium citrate (9:1, v/v) to obtain platelet-poor plasma, samples were centrifuged twice at $2000 \mathrm{~g}$ for 15 minutes for the determination of PT, PC and INR (using Siemens coagulometer, Germany) and for VWF-Ag assay plasma was separated aliquoted and stored at $-80^{\circ} \mathrm{C}$ till used and the rest were put in a plain vacutainer for determination of liver function and renal func- 
tion tests (using Cobas C311, Roche diagnostics).

VWF-Ag measurement: using TECHNOZYM ${ }^{\circledR}$ VWF-Ag ELISA; based sandwich technique, cat no 5450201 (Technoclone Vienna, Austria). Normal range for VWF-Ag is between $0.5-1.5 \mathrm{U} / \mathrm{ml}$ (50\% - 150\%).

\subsection{Ethical Approval}

Approval of the research was obtained from the Faculty Ethical Committee and written consent was obtained from each subject before the start.

\subsection{Statistical Analysis}

Data entry and analysis were done using statistical package of social science (SPSS) version 16 . The data are presented as means \pm SD and number, (\%). Statistical methods included independent t-tests and One-Way ANOVA test were used for comparison between groups. Logistic regression analysis was done to detect the independent predictors for statistically significant variables. ROC curve was performed to assess the value of VWF in predicting the presence of OVs and large varices. $\mathrm{P}$ value $<0.05$ was considered statistically significant.

\section{Results}

1) Demographic data for all patients: 62 patients with liver cirrhosis were recruited for the current study; 38 males and 24 females with their mean age $46 \pm 12$ years old. Jaundice was found in 8 (13\%) patients, history of haematemesis was detected in 13 (21\%) patients, hepatic encephalopathy in 12 (19.4\%) patients and ascites in 22 (35.5) patients (Table 1).

2) Characteristic endoscopic data for the studied patients: absence of OVs (NO) was found in 22 (35.5\%) patients and presence of OVs were found in 40 (64.5\%) patients; 12 (19.3\%) patients were G1, 14 (22.6\%) were G2 and 14 (22.6\%) were G3. Gastric varices were found in 5 (8\%) patients and portal hypertensive gastropathy in 40 (64.5\%) patients (Table 2).

3) Laboratory data for all patients: liver function tests including: serum bilirubin, total protein and albumin, liver enzymes (ALT and AST), prothrombin time and concentration, INR, alkaline phosphatase and platelet count in patients with and without varices are illustrated in Table 3. Only serum bilirubin was significantly higher in patients with varices than those without $(\mathrm{P}$ value $=0.04)$. No statistically significant difference was detected in the other parameters (Table 3). Child-Pugh score showed no significant difference between both groups so, plasma level of VWF-Ag not affected by this score in the current study.

Table 1. Demographic data for all studied patients.

\begin{tabular}{cc}
\hline Variable & Number (\%) (total = 62) \\
\hline Age & $46 \pm 12$ \\
Gender & \\
Male & $38(61.3)$ \\
Female & $24(38.7)$ \\
History & \\
Jaundice & $8(13)$ \\
Haematemesis & $13(21)$ \\
Encephalopathy & $12(19.4)$ \\
Ascites & $22(35.5)$ \\
Child-Pugh Class & \\
A & $30(48.4)$ \\
B & $20(32.2)$ \\
C & $12(19.4)$ \\
\hline
\end{tabular}

Data are expressed as number (\%) or mean $\pm \mathrm{SD}$. 
Table 2. Upper endoscopic data for all studied patients.

\begin{tabular}{cc}
\hline Variable & Number (\%) (total = 62) \\
\hline Esophageal varices & $22(35.5 \%)$ \\
No & $12(19.3 \%)$ \\
G1 & $14(22.6 \%)$ \\
G2 & $14(22.6 \%)$ \\
G3 & $5(8 \%)$ \\
Gastric varices & \\
Portal gastropathy & $40(64.5 \%)$ \\
Yes & $22(35.5 \%)$ \\
No &
\end{tabular}

Data are expressed as number, (\%).

Table 3. Laboratory data for patients with and without varices.

\begin{tabular}{cccc}
\hline Variable & No varices $(\mathrm{n}=22)$ & Varices $(\mathrm{n}=40)$ & P value \\
\hline Bilirubin (mg/dl) & $1.72 \pm 2.40$ & $2.18 \pm 2.78$ & 0.04 \\
ALT (IU/L) & $46.5 \pm 19.56$ & $64.30 \pm 50.32$ & NS \\
AST (IU/L) & $49.4 \pm 37.564$ & $80.20 \pm 54.35$ & NS \\
Albumin (g/dl) & $3.10 \pm 0.89$ & $2.43 \pm 0.66$ & NS \\
Protein (g/dl) & $6.76 \pm 1.2$ & $6.25 \pm 1.1$ & NS \\
PT(sec) & $14.32 \pm 3.43$ & $16.50 \pm 2.45$ & NS \\
PC (\%) & $63.16 \pm 13.32$ & $57.6 \pm 12.7$ & NS \\
INR & $1.62 \pm 0.81$ & $1.44 \pm 0.22$ & NS \\
ALP (IU/L) & $92.4 \pm 26.89$ & $122.13 \pm 79.36$ & NS \\
Platelets (cell/mm $\left.{ }^{3}\right)$ & $155.5 \pm 109.52$ & $115.13 \pm 78.87$ & NS \\
Child-Pugh score & $8.9 \pm 1.5$ & $9.5 \pm 1.2$ & NS \\
\hline
\end{tabular}

Data are expressed as mean \pm SD. P value $<0.05$ = significant. NS: non-significant. Unpaired student's t test was used in analysis.

4) Plasma Von Willebrand factor-Ag level: It was significantly higher in patients with OVs than those without varices $(\mathrm{P}$ value $=0.000)$. Also, its level was significantly higher in patients with higher grade of OVs; G3 than those with G1 or G2 (P value = 0.000). Patients with large OVs including those with G2 and G3 showed statistically significantly higher values of VWF than those with small OVs (NO and G1) (P value $=0.000$ ) (Table 4) and (Figure 1). A statistically significant positive correlation was detected between the grade of OVs and Von Willebrand factor-Ag level (rho $=0.712$ and $\mathrm{P}$ value $=0.000$ ) regardless to the Child-Pugh class of the patient (Figure 2).

5) Plasma Von Willebrand factor-Ag level and prediction of the presence of OVs:

Detection of OVs: VWF was independent predictor for detecting the presence of OVs with good sensitivity (90\%), specificity (77.3\%) and accuracy (85.5\%) when its cutoff value was at 1.74 . ROC curve showed that AUC was 0.922 (Table 5) and (Figure 3).

Detection of large OVs: VWF was independent predictor for detecting the presence of large OVs with good sensitivity (91.2\%), specificity (85.7\%) and accuracy (88.7\%) when its cutoff value was at 2.16. ROC curve showed that AUC was 0.973 (Table 5) and (Figure 4). 
Table 4. VWF-Ag level according to presence, grade and size of varices.

\begin{tabular}{ccc}
\hline \multicolumn{2}{c}{ Von Willebrand factor-Ag level (U/ml) mean \pm SD $^{*}$} & P value \\
\hline $\begin{array}{c}\text { Esophageal varices } \\
\text { No }\end{array}$ & $1.272 \pm 0.378$ & 0.000 \\
Yes & $2.492 \pm 0.891$ & 0.000 \\
Grade of varices & $1.635 \pm 0.370$ & \\
G1 & $2.50 \pm 0.397$ & \\
G2 & $3.216 \pm 0.930$ & \\
G3 & & \\
Size of varices & $1.453 \pm 0.347$ & $2.858 \pm 0.663$ \\
Small (NO-G1) & & \\
Large (G2-G3) &
\end{tabular}

${ }^{*}$ Unpaired student's t-test and One way ANOVA test were used. P value $<0.05$ = significant.

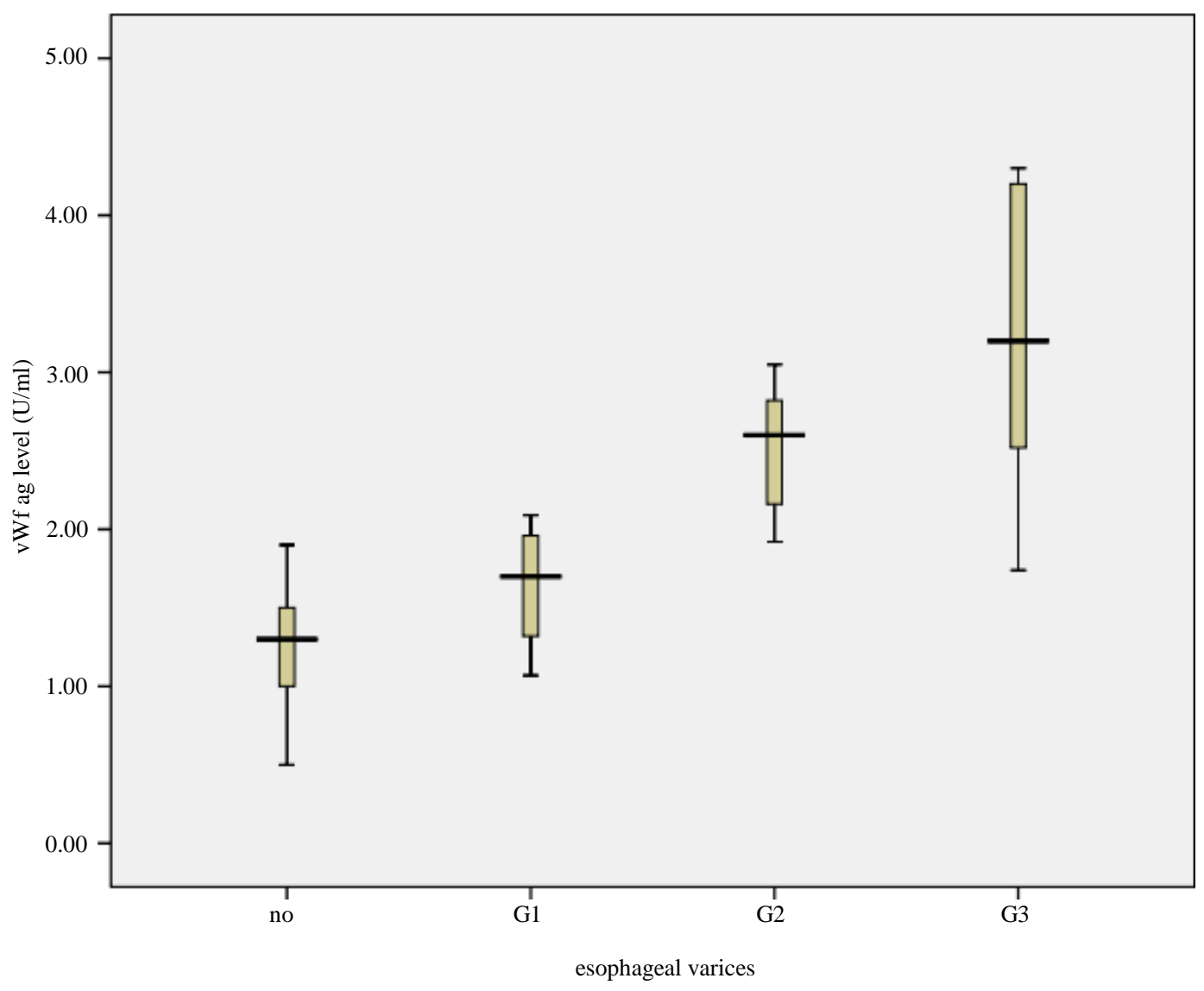

Figure 1. VWF level in patients with (G1, G2 and G3) and without esophageal varices.

\section{Discussion}

Ruptured OVs as a complication of portal hypertension is considered one of the major causes of death in cirrhotic patients and $30 \%$ of patients with liver cirrhosis will experience an episode of variceal hemorrhage within one year of diagnosis of varices [10]. 


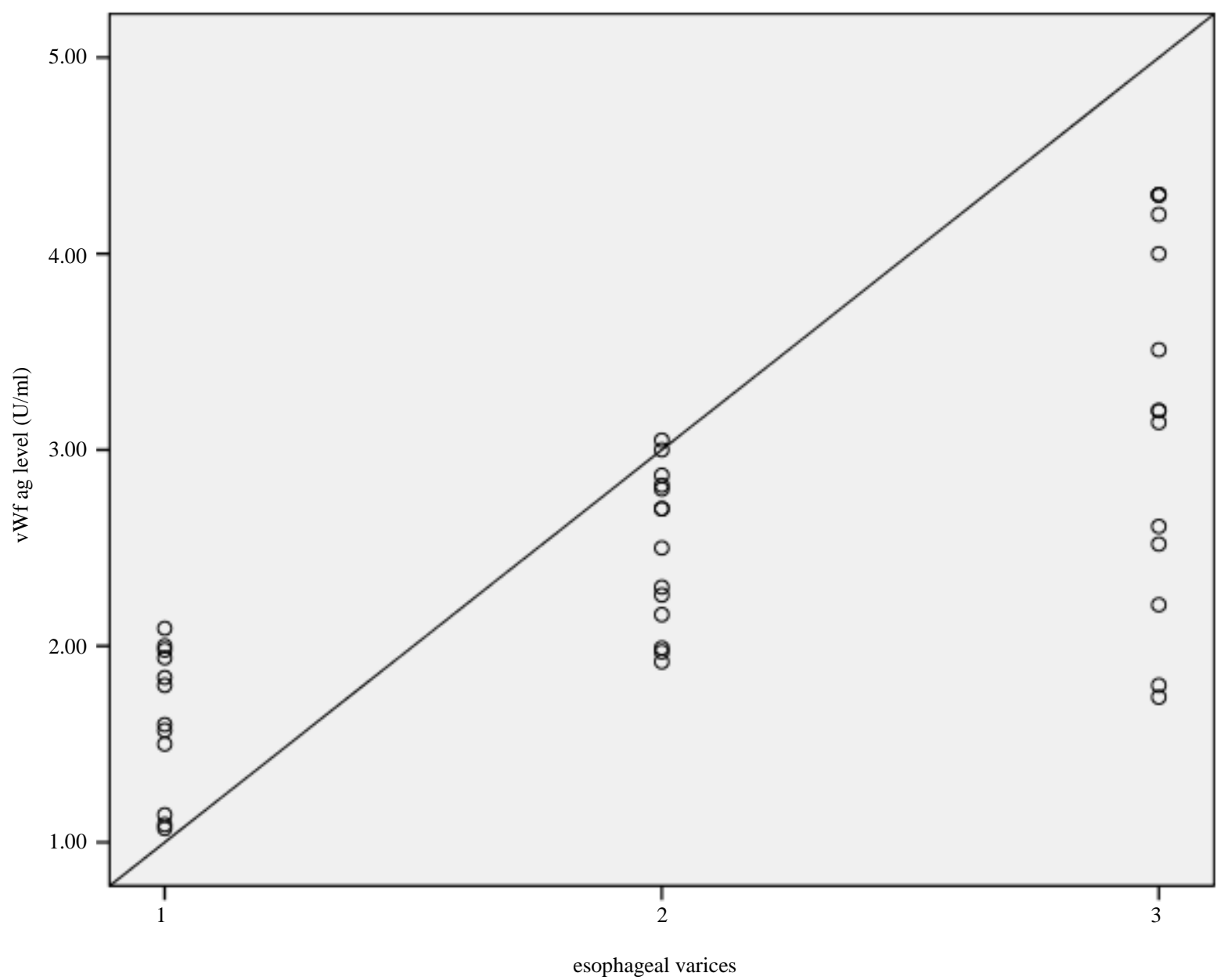

Figure 2. Correlation between the grade of esophageal varices and plasma Von Willebrand factor-Ag level (rho $=0.712$ and $\mathrm{P}$ value $=0.000)$.

Table 5. Logistic regression analysis for the Performance of VWF in prediction of presence of OVs and their sizes.

\begin{tabular}{cccccccc}
\hline VWF-Ag & Sensitivity & Specificity & Accuracy & P value & AUC & Cutoff value & Odds ratio \\
\hline Presence of varices & $90 \%$ & $77.3 \%$ & $85.5 \%$ & 0.000 & 0.922 & 1.74 & 0.452 \\
Presence of large varices & $91.2 \%$ & $85.7 \%$ & $88.7 \%$ & 0.000 & 0.973 & 2.16 & 0.383 \\
\hline
\end{tabular}

Different predictors for the detection of OVs with variable degrees of accuracy were evaluated; including laboratory and imaging techniques. Doppler US for hepatic hemodynamic also was evaluated in this field [11].

In the current study we evaluated the plasma level of VWF-Ag in patients with and without varices and in those with small and large varices to determine its possible role as a laboratory noninvasive marker in predicting the presence of varices and to get cutoff value for predicting those with large varices.

VWF-Ag was significantly higher in patients with OVs than those without varices and its level was significantly higher in patients with higher grade of OVs. The elevated levels of VWF in cirrhosis may be a consequence of endothelial perturbation, possibly caused by bacterial infection [12]. Another possible mechanism of elevated VWF in cirrhosis is induction of synthesis of VWF in the liver with cirrhosis itself [13], or reduced liver-mediated clearance resulting from decreased expression or activity of ADAMTS13 (VWF-Ag cleaving protease) [14] which may further increase VWF-Ag levels in patients with cirrhosis with PHT. Yilmaz et al. reported that plasma VWF-Ag level increase in cirrhotic patients and this is more pronounced with higher stages of cirrhosis according to Child-Pugh score [7]. Wu et al. reported that the VWF is a noninvasive predictor of portal hypertension and OVs in patients with cirrhosis and increased levels of VWF in liver tissues may induce 


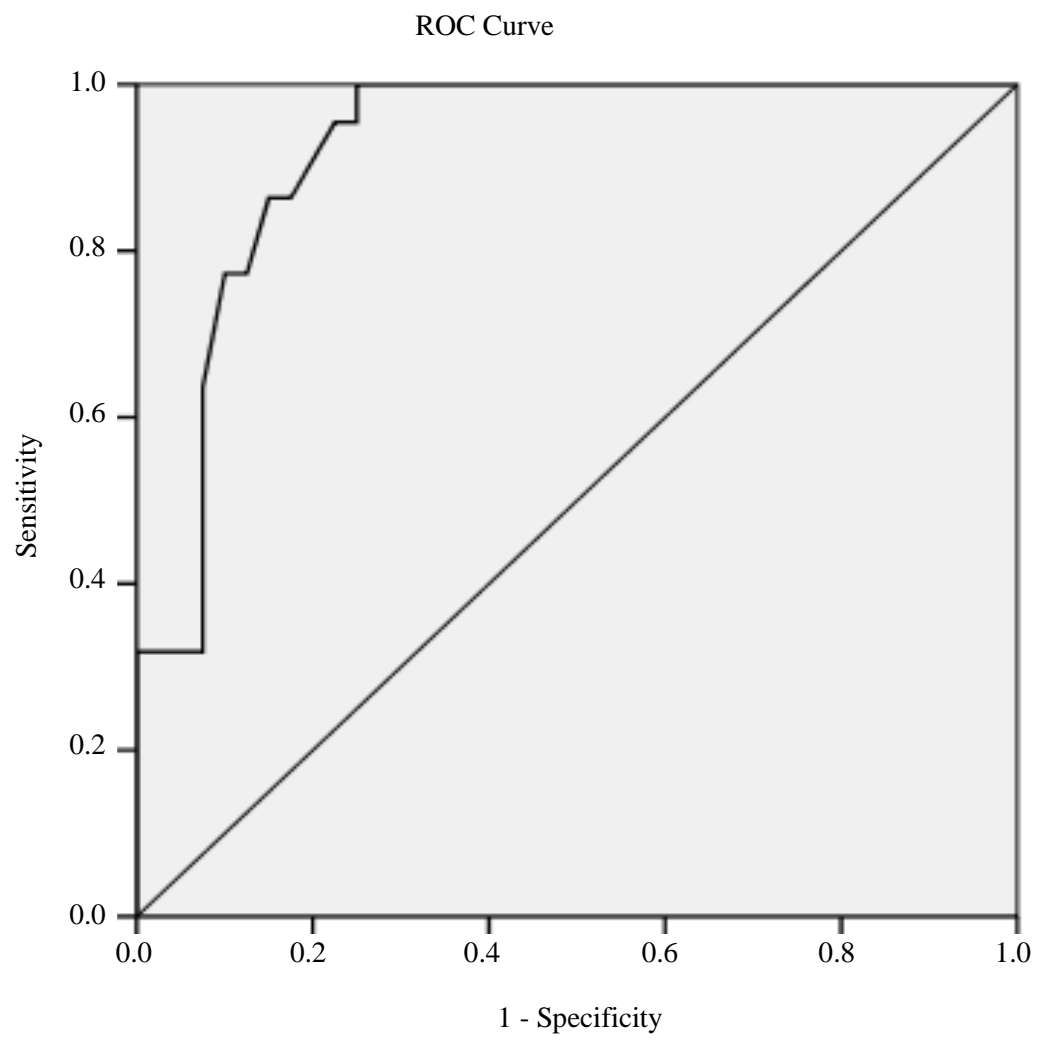

Figure 3. ROC curve for VWF in predicting the presence of OVs, AUC $=0.922$.

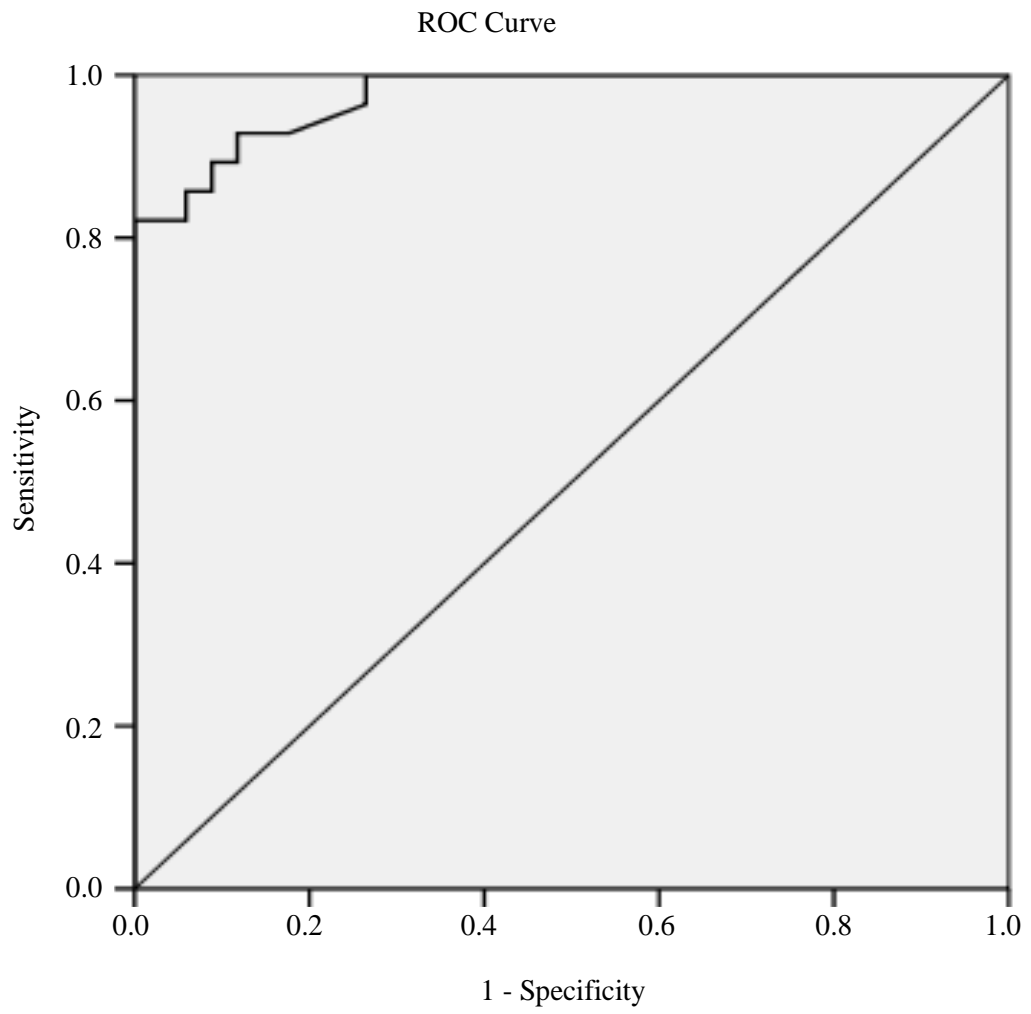

Figure 4. ROC curve for VWF in predicting the presence of large OVs, AUC $=0.973$. 
the elevated plasma VWF levels [15].

Also we found that patients with large OVs showed significant higher values of VWF than those with small varices. Ferlitsch et al. reported that VWF-Ag correlated with HVPG $(\mathrm{r}=0.69$; $\mathrm{P}<0.0001)$ and predicted clinically significant portal hypertension independently of Child Pugh score and higher VWF-Ag levels were associated with varices [8]. Ghweil et al. reported that VWF-Ag level was higher in patients with bleeding varices than those without bleeding and its value was independent predictor of esophageal variceal bleeding [16].

In the current study the cutoff value of VWF for the presence of OVs was $1.74 \mathrm{U} / \mathrm{ml}$. So, any cirrhotic patient with increased VWF value higher than that value should be screened by endoscopy for detecting the presence of OVs. Our results showed that the cutoff value of VWF-Ag for detecting large varices was $2.16 \mathrm{U} / \mathrm{ml}$. So, the priority of urgent endoscopic intervention for cirrhotic patients presenting with haematemesis should be directed to those with value higher than $2.16 \mathrm{U} / \mathrm{ml}$. The use of this marker might be of great value especially in endemic areas with large number of patients admitted with haematemesis attack and the limited number of endoscopic units with low facilities.

The limitation of this study was the inability to measure the hepatic venous pressure gradient as it is an invasive maneuver and not available in our locality.

\section{Conclusion}

VWF-Ag could be used as a non invasive laboratory independent predictor for the detection of OVs which may help in reducing the number of unnecessary endoscopies.

\section{Conflict of Interest}

Authors have declared that no competing interests exist.

\section{References}

[1] Bosch, J. and García-Pagán, J.C. (2000) Complications of Cirrhosis. I. Portal Hypertension. Journal of Hepatology, 32, 141-156. http://dx.doi.org/10.1016/S0168-8278(00)80422-5

[2] Arguedas, M.R., Heudebert, G.R., Eloubeidi, M.A., Abrams, G. and Fallon, M.B. (2002) Cost-Effectiveness of Screening, Surveillance, and Primary Prophylaxis Strategies for Esophageal Varices. American Journal of Gastroenterology, 97, 2441-2452. http://dx.doi.org/10.1111/j.1572-0241.2002.06000.x

[3] La Mura, V., Reverter, J.C., Flores-Arroyo, A., et al. (2011) Von Willebrand Factor Levels Predict Clinical Outcome in Patients with Cirrhosis and Portal Hypertension. Gut, 60, 1133-1138. http://dx.doi.org/10.1136/gut.2010.235689

[4] Sadler, J.E., Mannucci, P.M., Berntorp, E., Bochkov, N., Boulyjenkov, V., Ginsburg, D., et al. (2000) Impact, Diagnosis and Treatment of Von Willebrand Disease. Thrombosis and Haemostasis, 84, 160-174.

[5] Reiner, A.P., Siscovick, D.S. and Rosendaal, F.R. (2001) Hemostatic Risk Factors and Arterial Thrombotic Disease. Thrombosis and Haemostasis, 85, 584-595.

[6] Wanless, I.R., Wong, F., Blendis, L.M., Greig, P., Heathcote, E.J. and Levy, G. (1995) Hepatic and Portal Vein Thrombosis in Cirrhosis: Possible Role in Development of Parenchymal Extinction and Portal Hypertension. Hepatology, 21, 1238-1247.

[7] Yilmaz, V.T., Dincer, D., Avci, A.B. and Cetinkaya, R. (2015) Significant Association between Serum Levels of Von Willebrand Factor (vWF) Antigen with Stages of Cirrhosis. Eurasian Journal of Medicine, 47, 21-25. http://dx.doi.org/10.5152/eajm.2014.0016

[8] Ferlitsch, M., Reiberger, T., Hoke, M., Salzl, P., Schwengerer, B., Ulbrich, G., Payer, B.A., Trauner, M., Peck-Radosavljevic, M. and Ferlitsch, A. (2012) Von Willebrand Factor as New Noninvasive Predictor of Portal Hypertension, Decompensation and Mortality in Patients With Liver Cirrhosis. Hepatology, 56, 1439-1447. http://dx.doi.org/10.1002/hep.25806

[9] Beppu, K., Inokuchi, K., Koyanagi, N., et al. (1981) Prediction of Variceal Haemorrhage by Oesophageal Endoscopy. Gastrointestinal Endoscopy, 27, 213-218. http://dx.doi.org/10.1016/S0016-5107(81)73224-3

[10] Paunescu, V., Grigorean, V. and Popescu, C. (2004) Risk Factors for Immediate Outcome of Gastrointestinal Bleeding in Patients with Cirrhosis. Chirurgia [Bucur], 99, 311-322.

[11] Mahmoud, H.S., Mostafa, E.F. and Mohammed, M.A. (2014) Role of Portal Haemodynamic Parameters in Prediction of Oesophageal Varices in Cirrhotic Patients. Arab Journal of Gastroenterology, 15, 130-134.

http://dx.doi.org/10.1016/j.ajg.2014.09.001 
[12] Ferro, D., Quintarelli, C., Lattuada, A., Leo, R., Alessandroni, M., Mannucci, P.M., et al. (1996) High Plasma Levels of Von Willebrand Factor as a Marker of Endothelial Perturbation in Cirrhosis: Relationship to Endotoxemia. Hepatology, 23, 1377-1383. http://dx.doi.org/10.1002/hep.510230613

[13] Hollestelle, M.J., Geertzen, H.G., Straatsburg, I.H., van Gulik, T.M. and van Mourik, J.A. (2004) Factor VIII Expression in Liver Disease. Thrombosis and Haemostasis, 91, 267-275.

[14] Lisman, T., Bongers, T.N., Adelmeijer, J., Janssen, H.L., de Maat, M.P., de Groot, P.G. and Leebeek, F.W. (2006) Elevated Levels of Von Willebrand Factor in Cirrhosis Support Platelet Adhesion despite Reduced Functional Capacity. Hepatology, 44, 53-61. http://dx.doi.org/10.1002/hep.21231

[15] Wu, H., Yan, S., Wang, G., Cui, S., Zhang, C. and Zhu, Q. (2015) Von Willebrand Factor as a Novel Noninvasive Predictor of Portal Hypertension and Esophageal Varices in Hepatitis B Patients with Cirrhosis. Scandinavian Journal of Gastroenterology, 16, 1-10. http://dx.doi.org/10.3109/00365521.2015.1037346

[16] Ghweil, A.A., Arafa, U.A., Khodeary, A. and Salem, A.N. (2014) Predictors of Bleeding from Esophageal Varices: The Role of Factor VII and Von Willebrand Factor (vWF). Open Journal of Gastroenterology, 4, 152-158. http://dx.doi.org/10.4236/ojgas.2014.44023

\section{Abbreviations}

VWF: Von Willebrand Factor;

VWF-Ag: Von Willebrand Factor-Antigen;

OVs: Esophageal Varices;

PHT: Portal Hypertension;

PHG: Portal Hypertensive Gastropathy. 